\title{
Etude expérimentale d'un nouveau sulfamide hypoglycémiant particulièrement actif, le HB 419 ou glibenclamide*
}

\author{
II. Action bêtacytotrophe et stimulatrice de la néogénèse des îlots de Langerhans
}

\author{
A. Loubatières, M.M. Mariani, R. Alrio, G. Ribes, H. de Malbosc et M.H. Houareau
}

Reçu le 4 janvier, 1969

Experimental study on glibenclamide (HB 419), a new particularly active hypoglycaemic sulphonamide. II. Betacytotrophic action and stimulation of the neogenesis of the islets of Langerhans.

Summary. The authors have demonstrated that HB 419 chronically administered in the mouse, stimulated the development of the islets of Langerhans and particularly that of the beta cells. This substance is thus endowed with a "beta eytotrophic" action. - In the normal dog, HB 419 administered over a four-month period resulted in a decreased fasting glycaemia, as well as a decrease of the entire nyctohemeral glycaemia curve. The experiments carried out showed that under the influence of prolonged HB 419 administration, the amount of endogenous insulin synthesized and secreted over and above the normal secretory level, was considerable.

Résumé. Les auteurs ont montré chez la souris que le HB 419 administré chroniquement, stimule le développement des îlots de Langerhans et des cellules bêta. Ce produit est done doué de l'kaction bêtacytotrophe». Chez lo chien normal, l'administration pendant 4 mois de HB 419 a pour effets d'abaisser la glycémie à jeun ainsi que l'ensemble de la courbe de la glycémie nycthémérale. - L'expérimentation effectuée montre que sous l'influence de l'administration prolongée de HB 419 , la quantité d'insuline endogène synthétisée et sécrétée en plus de la sécrétion normale est considérable.

Experimentelle Untersuchungen an einem besonders stark blutzuckersenkenden Sulfonamid, dem HB 419 oder Glybenclamid. II. Betacytotrophe Wirkung und Stimulierung der Neubildung von Langerhans'schen Inseln.

Zusammenfassung. Die Autoren zeigten an Mäusen, daß chronische Gaben von HB 419 die Entwicklung der Langerhans'schen Inseln und der B-Zellen fördern. Die Substanz besitzt also eine, ,betacytotrophe Wirkung ${ }^{\text {.c }}$. Bei normalen Hunden bewirkt Zufuhr von HB 419 über 4 Monate eine Senkung der Nüchternblutzucker-Werte und der 24 Std-Blutzuckerkurven. Unter den gewählten Versuchsbedingungen konnte demonstriert werden, daß es unter dem Einfluß einer Dauerzufuhr von HB $419 \mathrm{zu}$ einer erheblichen Mehrproduktion und Mehrfreisetzung von endogenem Insulin kommt.

Key-words: Glibenclamide, hypoglycaemic sulphonamide, oral antidiabetic substances, islets of Langerhans, islet development, beta cells, betacytotrophic action, insulin secretion, insulin synthesis, diabetes prevention.
Nous avons rapporté dans de précédentes publications $[34,40,41]$ les conclusions des expériences poursuivies dans notre laboratoire en vue de mettre en évidence le mécanisme d'action d'un sulfamide hypoglycémiant particulièrement actif le $\mathrm{N}-(4-(\beta-<-2-$ méthoxy-5-chlor-benzamido >-éthyl)-benzène-sulfonyl)-N'-cyclohexyl-urée ou glibenclamide ou HB 419. Rappelons que cette substance possède les actions essentielles suivantes:

1. Elle stimule in vitro (sur le pancréas isolé et perfusé du rat) et in vivo (sur le chien normal) la sécrétion d'insuline endogène.

2 Elle détermine chez l'animal entier une hypoglycémie profonde et durable qui peut se poursuivre pendant $24 \mathrm{~h}$ et est concomitante d.une sécrétion prolongée d'insuline.

3. Elle ne manifeste pas son action hypoglycémiante chez l'animal totalement dépancréaté. Cette action dépend done de la présence dans l'organisme de tissu pancréatique normal, renfermant des cellules bêta normales.

* Le HB 419 ou glibenclamide portait initialement le nom de glybenzcyclamide. La dénomination commune internationale qui vient d'être définitivement adoptée est glibenclamide.
4. Le HB 419 potentialise chez le chien totalement dépancréaté les effets hypoglycémiants propres d'une dose déterminée d'insuline.

5. Comme toutes ces actions se manifestent à des doses très inférieures à celles qui sont nécessaires pour obtenir les mêmes effets à l'aide d'autres sulfamides hypoglycémiants, le glibenclamide apparaît comme le sulfamide hypoglycémiant le plus actif.

Il demeurait cependant à établir si, à l'instar des autres sulfamides hypoglycémiants jusqu'ici étudiés, le glibenclamide possédait la propriété de provoquer le développement des îlots de Langerhans et en particulier celui des cellules bêta insulino-sécrétrices (action bêtacytotrophique) [24].

Rappelons qu'en 1946 furent décrits pour la première fois les effets d'un sulfamide hypoglycémiant, le para-amino-benzène-sulfamido-isopropyl-thiodiazol (2254 RP) sur les îlots de Langerhans du pancréas du lapin (Lodbatik̀res [19], p. 78). Après vingt jours d'administration «les îlots de Langerhans ont été trouvés hyperplasiques. Les cellules étaient augmentées de volume et leurs noyaux étaient également de dimensions supranormales. II s'agissait d'éléments en pleine activité ne présentant aucune manifestation dégénératrice». D'autre part, il a été reconnu que ce sulfamide n'était pas «doué d'une action toxique pour 
le tissu endocrinien du pancréas, puisque, dans certaines conditions tout au moins, il était possible de reconnaître à ce corps une influence plutôt trophique».

Par la suite, la stimulation de la néogénèse des cellules bêta provoquée par divers sulfamides hypoglycémiants dérivés du thiodiazol (notamment le paraamino-benzène-sulfamido-tertiobutyl-thiodiazol) ou de l'urée (tolbutamide, chlorpropamide, azépinamide) a été constatée histologiquement chez le lapin $[19,26]$, le chion $[30,8]$, le rat $[43,3,6,9,15,14,31,45]$, le hamster [46], le chat [17] la souris [5].

L'augmentation du poids des îlots de Langerhans a été observée sur le rat normal $[2,7,31,45,35]$ ou sur le rat hypophysectomisé [7]. En outre des mitoses ont été observées chez les rats $[15,14]$ ou le chien [30] traités par les sulfamides.

Chez l'homme, il a été constaté que le traitement sulfamidé provoque l'hyperplasie insulaire $[9,4,18]$.

En outre l'action stimulatrice des sulfamides hypoglycémiants sur le développement des îlots a été observée sur des cultures de pancréas foetal du rat $[10$, $47,48,16]$, des transplants de tissu pancréatique dans la chambre antérieure de l'oeil du rat [10] ou bien dans des homogreffes de tissu pancréatique néonatal dans la poche jugale du hamster doré [46].

Cette action néogénétique que les sulfamides hypoglycémiants exercent sur les cellules bêta des îlots de Langerhans a été utilisée pour provoquer la guérison de divers types de diabètes expérimentaux d'intensité modérée notamment le diabète méta-alloxanique du lapin et du chien $[19,26,28,20,43,27,29]$, ou le diabète métahypophysaire du chat [17]. M a été d'autre part démontré que les sulfamides hypoglycémiants sont capables de retarder ou de prévenir l'installation du diabète sucré chez les chiens privés des neuf-dixièmes du pancréas $[36,37,25,39,33]$.

C'est sur ces résultats expérimentaux satisfaisants qu'a été proposée pour la première fois l'utilisation des sulfamides hypogylcémiants "comme agents prophylactiques et retardateurs du diabète chez les sujets prédisposés à cette affection》 [21].

Nos expériences concernant l'action bêtacytotrophe éventuelle du glibenclamide ont été divisées en deux groupes :

1. Mise en évidence de l'hyperplasie insulaire et mise en évidence éventuelle d'une action stimulatrice sur la néogénèse des cellules bêta chez l'animal normal (souris et rat).

2. Recherche des conséquences de l'action bêtacytotrophique exercée par le glibenclamide sur la glycémie et la sécrétion d'insuline du chien normal.

I. Hyperplasie des îlots dé Langerhans et néogénèse des cellules bêta chez l'animal normal (souris et rat)

\section{Etude quantitative.}

Nos expériences ont été réalisées sur deux lots de souris Swiss issues de notre élevage; le premier était constitué par 29 souris de sexe femelle, de poids initial compris entre 15 et $20 \mathrm{~g}$, (lot A) (souris jeunes); le deuxième par 31 souris de sexe mâle dont le poids initial se situait entre 30 et $35 \mathrm{~g}$, (lot B) (souris adultes). Elles recevaient un régime standard complet équilibré et de l'eau de boisson à volonté. Chaque lot a été divisé en trois groupes: Un groupe de souris témoins et deux groupes de souris traitées par le HB 419 à deux doses différentes.

Les doses de HB 419 administrées chaque jour ont été de $2 \mathrm{mg} / \mathrm{kg}$ et $5 \mathrm{mg} / \mathrm{kg}$ pour les souris du premier lot, et de $5 \mathrm{mg} / \mathrm{kg}$ et $125 \mathrm{mg} / \mathrm{kg}$ pour celles du deuxième lot. Le produit a été administré en suspension dans une solution de gomme adragante à $0.5 \%$, par voie digestive, à l'aide d'une sonde oesophagienne. Les animaux témoins n'ont reçu que la solution de gomme adragante. La quantité de solution administrée dans tous les cas (témoins et traités) a été de $1 \mathrm{ml}$ pour $100 \mathrm{~g}$ de poids. La durée de l'expérience a été de 35 jours.

Le prélèvement du pancréas a été effectué pour tous les animaux $24 \mathrm{~h}$ après la dernière administration de produit, sous anesthésie au nembutal. Afin de colorer les îlots de Langerhàns sur l'animal vivant, selon la technique de HaIST et PUGH [12], une solution de rouge neutre à $2.5 \%$ dans du sérum physiologique était d'abord injectée dans le veine cave inférieure $(1 \mathrm{ml}$ pour $100 \mathrm{~g}$ de poids). Le pancréas était prélevé dans sa totalité $15 \mathrm{~min}$ après l'injection et pesé. Ensuite, il était placé dans un récipient, maintenu à la température de la glace fondante et découpé en petits fragments. Ceux-ci montés entre deux lames de verre maintenues par des pinces, ont été congelés rapidement dans un mélange de neige carbonique et d'alcool. Les préparations ont été ensuite conservées au congélateur à $-30^{\circ} \mathrm{C}$ jusqu'au moment de leur projection. Les lames ont été alors ramenées à la température de la pièce et les images projetées ont été dessinées puis soigneusement planimétrées. Les surfaces respectives des îlots et du pancréas total ont été ensuite calculées après correction par les grandissements utilisés. Ceux-ci étaient: $100 \times$ pour les îlots et $5 \times$ pour le pancréas total.

Nous avons alors déterminé le rapport: surface îlots

surface totale du pancréas qui est proprotionnel au rapport

$\frac{\text { volume îlots }}{\text { volume total du pancréas }}$

comme l'ont établi Haist et Pugh [12], pour le rat. Le poids total du pancréas étant d'autre part connu, nous avons calculé la valeur suivante:

surface îlots $\overline{\text { surface totale du pancréas }} \times$ poids du pancréas en $\mathrm{mg}$,

que nous avons dénommée index insulaire et qui est directement proportionnelle au poids des îlots; c'est cette valeur qui a été utilisée pour tous nos calculs statistiques. Pour obtenir le poids réel du tissu insulaire il convient de multiplier l'index insulaire par un facteur de correction qui est le rapport:

épaisseur moyenne des îlots

épaisseur moyenne des préparations 
HaIst et Pugri ont déterminé expérimentalement ce rapport chez le rat et l'ont trouvé égal à 0.4. Dans notre travail il ne nous a pas paru nécessaire d'établir la valeur de ce rapport chez la souris; notre but étant de comparer le développement du tissu insulaire chez des souris traitées et chez des souris témoins, cette comparaison pouvait s'effectuer en utilisant les index insulaires.

Dans le Tableau 1 sont rapportés les résultats numériques enregistrés avec les deux lots de souris. Les index insulaires sont donnés d'abord en valeur absolue, puis rapportés à $100 \mathrm{~g}$ de poids corporel et à $100 \mathrm{mg}$ de pancréas. gonales suivantes: animaux traités par rapport au groupe témoin, et groupes d'animaux traités entre eux.

De l'étude des résultats obtenus avec le lot $\mathrm{A}$ il ressort que l'administration chronique de glibenclamide par voie orale, aux doses quotidiennes de 2 $\mathrm{mg} / \mathrm{kg}$ et de $5 \mathrm{mg} / \mathrm{kg}$, augmente d'une manière indiscutable en 35 jours, le poids des îlots; cette augmentation est respectivement de $32,5 \%$ et $25 \%$. Comme le montre la figure, le groupe d'animaux traités diffère significativement des témoins $(p<0.025)$; d'autre part, il n'apparaît pas de différence significative entre les comportements des deux groupes d'animaux traités par les deux doses de $2 \mathrm{mg} / \mathrm{kg}$ et $5 \mathrm{mg} / \mathrm{kg}$.

Tableau 1

\begin{tabular}{lllll}
\hline \multirow{2}{*}{ Souris } & \multicolumn{5}{l}{\begin{tabular}{l} 
Index insulaires \\
\cline { 2 - 5 }
\end{tabular}} & $\begin{array}{l}\text { Valeurs } \\
\text { absolues }\end{array}$ & $\begin{array}{l}\text { Pour } 100 \mathrm{~g} \text { de } \\
\text { poids corporel }\end{array}$ & $\begin{array}{l}\text { Pour } 100 \mathrm{mg} \\
\text { de pancréas }\end{array}$ \\
\hline \multirow{2}{*}{ Lot A } & témoins & $0.765 \pm 0.071$ & $3.716 \pm 0.385$ & $0.340 \pm 0.022$ \\
& HB $4192 \mathrm{mg} / \mathrm{kg}$ & $1.014 \pm 0.073$ & $4.494 \pm 0.245$ & $0.398 \pm 0.023$ \\
& HB $4195 \mathrm{mg} / \mathrm{kg}$ & $0.955 \pm 0.066$ & $4.979 \pm 0.359$ & $0.471 \pm 0.040$ \\
\hline \multirow{2}{*}{ Lot B } & témoins & $1.742 \pm 0.092$ & $5.471 \pm 0.327$ & $0.467 \pm 0.029$ \\
& HB $4195 \mathrm{mg} / \mathrm{kg}$ & $2.138 \pm 0.110$ & $7.129 \pm 0.652$ & $0.586 \pm 0.033$ \\
& HB $419125 \mathrm{mg} / \mathrm{kg}$ & $2.189 \pm 0.170$ & $6.210 \pm 0.546$ & $0.587 \pm 0.045$ \\
\hline
\end{tabular}

Sur la Fig. 1 sont représentées les modifications du poids des îlots que nous avons enregistrées avec les deux lots de souris A et B; elles sont exprimées en pourcentages par rapport aux témoins. Le poids corporel moyen mesuré en fin d'expérience est indiqué pour chaque groupe (témoins et traités). Les niveaux de significativité qui accompagnent nos résultats ont été établis au moyen de tests $\mathbf{F}$ pratiqués après analyse de variance. On a procédé aux comparaisons ortho-

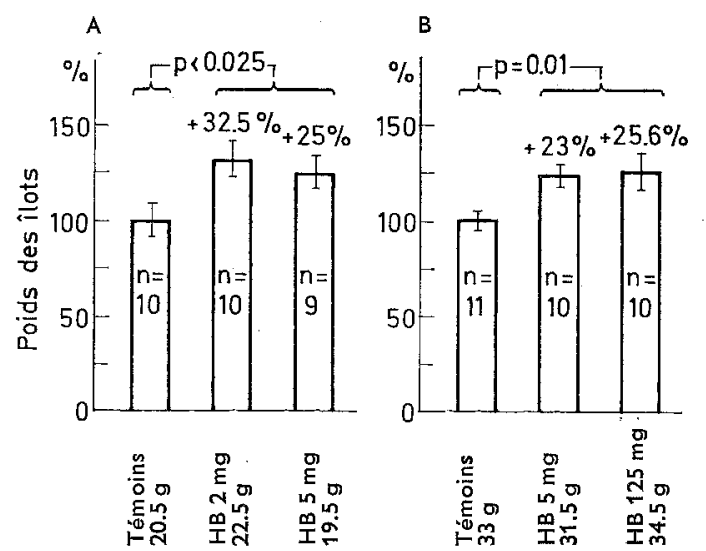

Fig. 1. Modification du poids des îlots chez deux lots de souris traitées par le glibenclamide (HB 419) à différentes doses. Les résultats sont évalués en pourcentages par rapport aux témoins. Chaque colonne représente la moyenne des poids des îlots \pm l'erreur standard de la moyenne calculée au sein du groupe. Le nombre d'animaux est indiqué pour chaque groupe dans la colonne correspondante et le poids corporel moyen au-dessous de la colonne.
En ce qui concerne le lot B, on voit que la dose de $5 \mathrm{mg} / \mathrm{kg}$ et par jour a provoqué une augmentation du poids des îlots de $23 \%$, résultat pratiquement identique à celui trouvé dans la première série d'expériences. et celle de $125 \mathrm{mg} / \mathrm{kg}$ et par jour une augmentation de $25.6 \%$. Le groupe des animaux traités est également, dans cette seconde série d'expériences, significativement différent des témoins $(p=0.01)$. D'autre part, comme dans la première série, il n'apparaît pas de différence significative entre les comportements des animaux traités respectivement par $5 \mathrm{mg} / \mathrm{kg}$ et $125 \mathrm{mg} / \mathrm{kg}$. L'augmentation importante de la dose à $125 \mathrm{mg} / \mathrm{kg}$ ne paraît donc pas avoir de pouvoir néoformateur sur l'appareil insulaire, supérieur à celui des doses plus faibles que nous avons utilisées, dans les conditions expérimentales où nous nous sommes placés.

D'après l'ensemble de ces résultats, le glibenclamide administré chroniquement, provoque une augmentation indiscutable de la masse du pancréas endocrine. Cet effet a été observé indifféremment sur les souris adultes et sur celles en voie de croissance, ainsi que sur les souris mâles et femelles. Il n'est, d'autre part, pas apparu de différences significatives entre les réponses aux trois doses utilisées: $2 \mathrm{mg} / \mathrm{kg}, 5 \mathrm{mg} / \mathrm{kg}$ et $125 \mathrm{mg} / \mathrm{kg}$.

Il s'avère donc que, de même que les sulfamides hypoglycémiants précédemment étudiés (glybuthiazol, tolbutamide, carbutamide, chlorpropamide, azépinamide), le glibenclamide (HB 419) s'est révélé doué de la propriété de stimuler la formation de tissu insulaire [38]. 


\section{Etude histologique}

Histologiquement l'action bêtacytotrophique des îlots s'est traduite chez les rats traités pendant 47 jours par la polynésie insulaire (augmentation du nombre des îlots) ainsi que par leur hyperplasie (augmentation de la surface des îlots). paraissait avoir disparu. A la fin du traitement, les cellules bêta étaient relativement riches en granules d'insuline Fig. 3. Ce fait suggère que, bien que chroniquement stimulées par le glibenclamide, les cellules bêta s'avèrent capables de synthétiser de l'insuline qui peut être libérée et jouer son rôle métabolique. Les

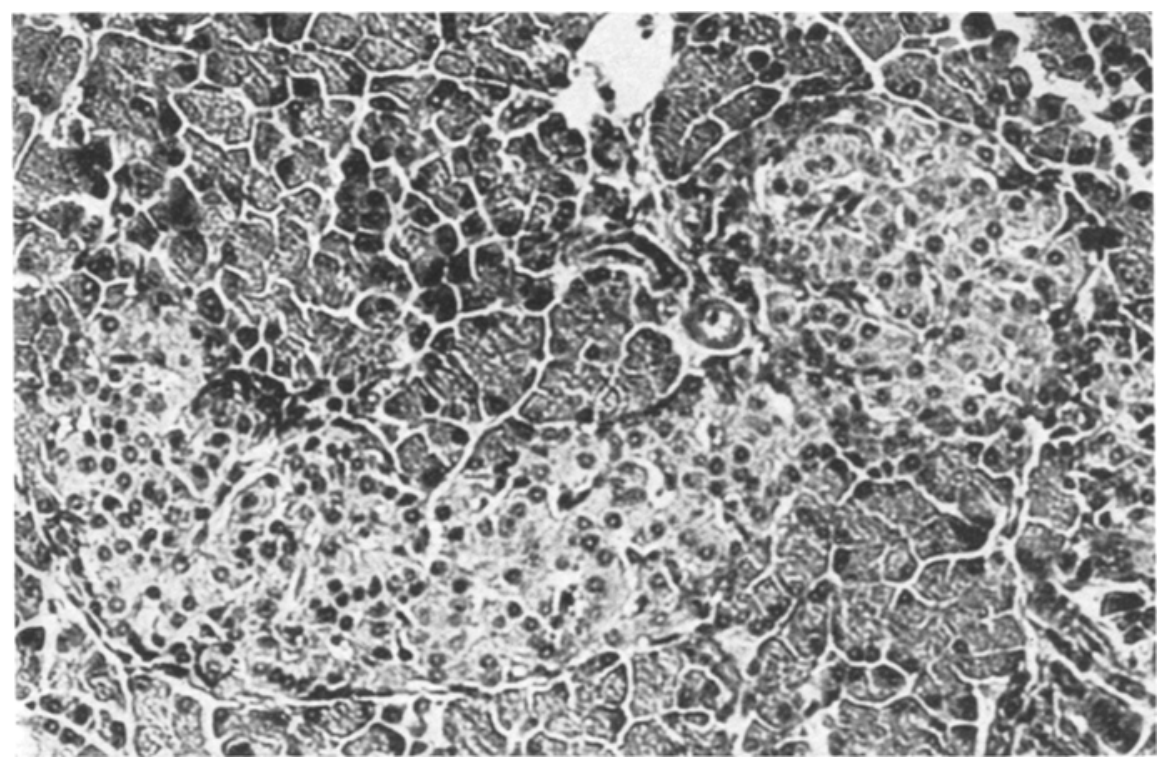

Fig. 2. Tlots de Langerhans hypertrophiés ot multilobés observés chez un rat de $200 \mathrm{~g}$ traité pendant 47 jours par HB 419 (125 mg/kg par jour, per os). - Coloration Heidenhaim Azan - Grossissement $\times 200$

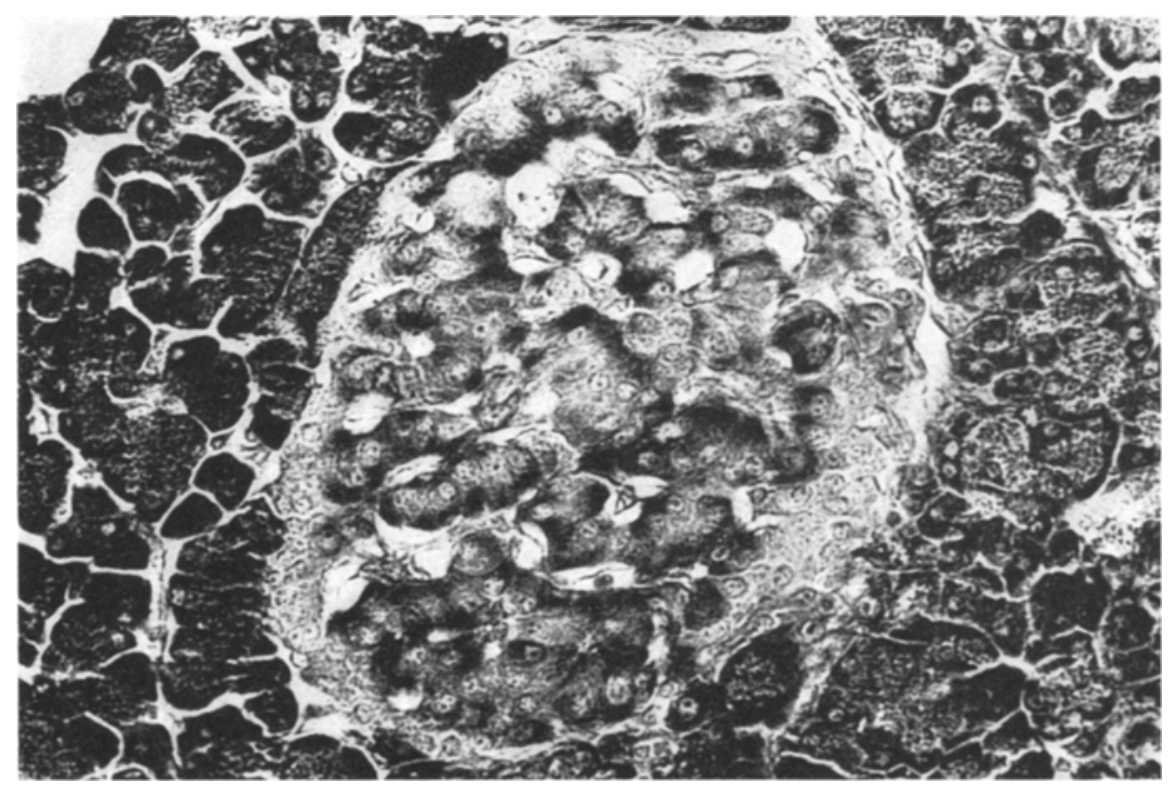

Fig. 3. Ilot de Langerhans observé chez le même rat traité pendant 47 jours par HB 419 (125 mg/kg par jour, per os). - Coloration Paraldéhyde-fuschine Grossissement $\times 500$. Noter la présence de nombreux granules d'insuline

Le développement d'îlots voisins juxtaposés donnait l'aspect d'îlots considérablement agrandis et multilobés Fig. 2. La limite conjonetive entre les îlots nouvelles cellules bêta naissaient aux dépens des cellules canaliculaires et des cellules indifférenciées (ou "cellules intermédiaires»). 
Ces constatations sont parfaitement en accord avec nos observations antérieures. Elles sont également en accord avec les observations de BuNNAG et WARNER (1966) [5] effectuées chez la souris traitée chroniquement par le tolbutamide ( $250 \mathrm{mg} / \mathrm{kg} /$ intrapéritonéal). Utilisant les méthodes radioautographiques, les auteurs ont constaté que le nombre des îlots augmentait $(+62 \%$ chez les traités pendant 4 mois par rapport aux témoins), que l'index radioactif et l'index mitotique s'élevaient considérablement dans les cellules canaliculaires et les cellules insulaires alors que les index des cellules acineuses ne subissaient pratiquement pas de modification.

Il convient de remarquer que l'excès d'insuline endogène dans le sang qui est produit par la stimulation des cellules bêta n'a pas déterminé la réduction de la croissance des îlots de Langerhans, comme le feraient d'après Haist les injections d'insuline exogène [11].

II. Action bêtacytotrophique exercée par le glibenclamide sur le pancréas du chien normal, envisagée du point de vue de la glycémie et de la sécrétion d'insuline

Nous nous sommes demandé si l'action bêtacytotrophique des sulfamides hypoglycémiants n'était pas seulement une expression morphologique et pondérale sur les îlots de Langerhans mais si elle se traduisait également par une manifestation fonctionnelle, telle que la synthèse et la libération d'une quantité accrue d'insuline endogène.

Trois chiens normaux adultes âgés de 2 ans, d'un poids de $15 \mathrm{~kg}$, en bonne santé, ont été soumis pendant 5 mois à l'administration quotidienne de glibenclamide (HB 419) à la dose de $2 \mathrm{mg}$ par $\mathrm{kg}$ per os et par jour. Leur régime alimentaire était constitué par $30 \mathrm{~g}$ de viande crue de cheval par kg et par jour, administrée en deux repas.

1. Des courbes nycthémérales de glycémie ont été établies au début et en cours de traitement afin d'étudier l'évolution de la glycémie après une prise orale de HB 419 (2 $\mathrm{mg} / \mathrm{kg}$ ), le dernier repas était administré la veille, 18 heures avant l'expérience. Les glycémies ont été dosées à l'aide de l'Auto Analyseur Technicon par la méthode au ferricyanure de potassium sur sang hémolysé [1]. Ces courbes sont représentées sur la Fig. 4. La courbe I a été enregistrée le premier jour de l'administration de glibenclamide. Les courbes II et III ont été enregistrées respectivement après un mois et quatre mois de traitement par le glibenclamide. Dans ces deux cas, la dernière administration de glibenclamide avait été faite la veille, $24 \mathrm{~h}$ avant le début de l'expérience.

De l'étude de ces courbes il ressort les faits suivants: - Lors de la première administration de HB 419 per os la glycémie n'est pas encore revenue à un taux normal $24 \mathrm{~h}$ après la prise, ce qui est compatible avec le maintien d'une hypersécrétion d'insuline $24 \mathrm{~h}$ après l'administration.
- Par la suite et au fur et à mesure que le traitement par le HB 419 se prolonge, la glycémie à jeun s'abaisse ainsi que le niveau de la glycémie considéré tout au long de la journée. Après 4 mois de traitement la courbe nycthémérale de la glycémie demeure basse pendant les $24 \mathrm{~h}$, réalisant pratiquement un plateau dont le niveau moyen se situe autour de $0.55 \mathrm{~g}$ de glucose par litre de sang.

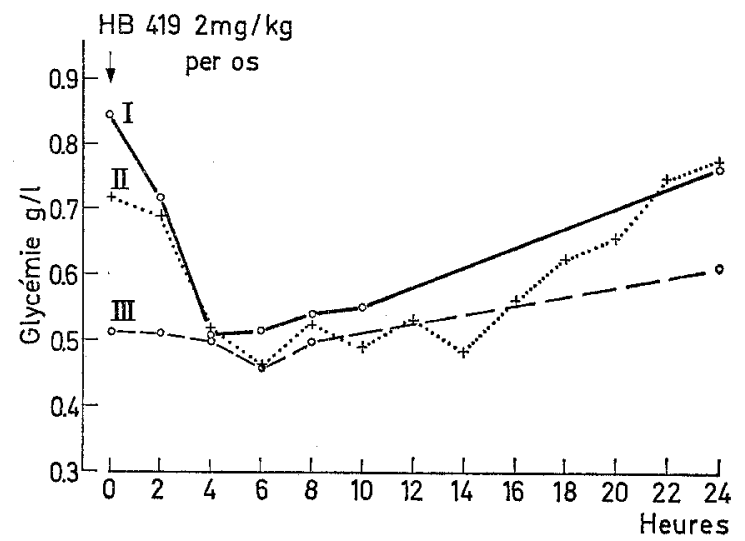

Fig. 4. Evolution nycthémérale de la glycémie chez le chien éveillé après une prise orale de HB 419 (2 $\mathrm{mg} / \mathrm{kg})$. Chaque courbe correspond à la moyenne des valeurs obtenues chez 3 chiens. La courbe I a été enregistrée lors de la première administration de HB 419. Les courbes II et III ont été enregistrées chez les mêmes chiens après un traitement respectivement de 1 mois et de 4 mois par le HB 419

Diverses hypothèses peuvent être proposées pour expliquer l'abaissement progressif de la glycémie initiale à jeun constaté au cours de la prolongation du traitement ainsi que celui du niveau de la glycémie considéré tout au long de la journée.

1. Il peut s'agir, en ce qui concerne le taux de la glycémie initiale à jeun, du reliquat de l'action hypoglycémiante de la dose de HB 419 administrée $24 \mathrm{~h}$ auparavant et qui peut se prolonger au delà de cette durée. Cependant cette explication n'est que partiellement satisfaisante car alors, on ne comprendrait pas pourquoi l'abaissement glycémique observé après 4 mois de traitement par le sulfamide est supérieur à celui constaté après un mois de traitement.

2. Il peut s'agir d'une fixation et d'une accumulation de HB 419 dans le tissu réticulo endothélial. Le sulfamide se trouverait par la suite libéré et exercerait son action hypoglycémiante qui s'ajouterait à celle du HB 419 récemment administré. Cette hypothèse n'arrive pas cependant à nous convaincre totalement.

3. Il peut s'agir d'une modification du seuil de sensibilité de la cellule bêta soit au glucose, soịt au HB 419 . S'il en était ainsi, la cellule réagirait au sulfamide (pour des concentrations de glucose ou de HB 419 plus basses) en sécrétant davantage d'insuline qu'une cellule bêta normale.

4. I peut s'agir d'un accroissement du nombre des cellules bêta sous l'effet de l'action bêtacytotrophique 
du HB 419, en particulier pour la courbe obtenue après 4 mois de traitement. Ces cellules sécrèteraient davantage d'insuline provoquant ainsi la baisse de la glycémie. C'est l'hypothèse qui nous paraît la plus vraisemblable. Il convient de remarquer toutefois que $24 \mathrm{~h}$ après la dernière administration du produit, l'animal est encore sous l'influence du sulfamide qui stimule les cellules bêta.

5. Il peut s'agir d'une addition des quatre effets précédents envisagés comme des hypothèses.

6. Il peut s'agir d'une augmentation de sensibilité sous l'effet du traitement chronique sulfamidé des cellules extrapancréatiques (cellules hépatiques, cellules adipeuses, cellules musculaires) à l'insuline sécrétée et circulant dans le torrent circulatoire. Cette hypothèse demeure cependant entièrement à démontrer.

7. II pourrait s'agir également de l'addition des effets suggérés dans les paragraphes $1,2,3,4$, et 6 .

Toutes ces éventualités sont actuellement à l'étude dans notre laboratoire.

2. Nous avons étudié d'autre part le comportement non seulement de la glycémie mais aussi celui de l'insulinémie à jeun, d'abord au cours d'une période de référence, préalable à tout traitement, ensuite à des périodes de temps variables, en cours de traitement, 10 jours, 1 mois, 4 mois. Les prélèvements de sang étaient effectués dans la veine jugulaire $18 \mathrm{~h}$ après le dernier repas et $24 \mathrm{~h}$ après la dernière prise de glibenclamide. L'insuline était dosée dans le plasma par la méthode radioimmunologique $B$ de HaLES et RANDLE [13].

La figure 5 représente les résultats obtenus. Chaque point représente la moyenne des valeurs mesurées chez les trois chiens pendant deux jours consécutifs. On constate que le taux de l'TRI plasmatique s'élève progressivement en même temps que la glycémie s'abaisse.

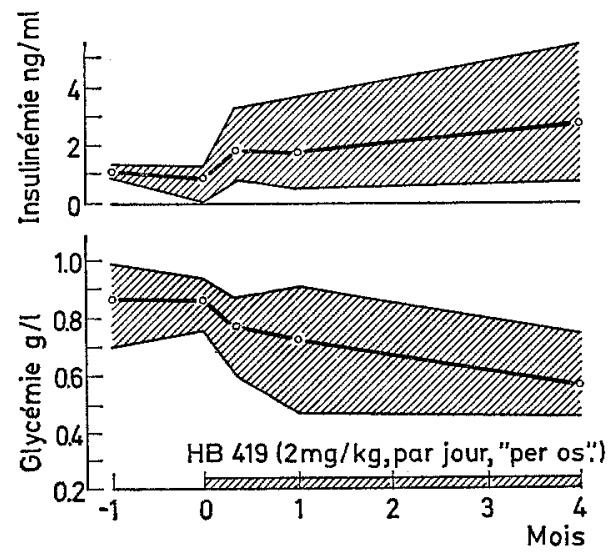

Fig. 5. Variations de la glycémie et de l'insulinémie chez le chien traité chroniquement par lo glibenclamide (HB 419) per os, à la dose de $2 \mathrm{mg} / \mathrm{kg}$ et par jour. Chaque point représente la moyenne des valeurs obtenues chez trois chiens durant deux jours consécutifs; la zone hachurée indique l'étendue des variations
Ce fait suggère donc que l'augmentation progressive de l'aire d'hypoglycémie des courbes nycthémérales en cours de traitement (Fig. 4) est due à l'augmentation de la quantité d'insuline présente dans le sang par rapport à la période de référence. (L'aire d'hypoglycémie est représentée par la surface comprise entre la courbe enregistrée sous l'action du HB 419 et une courbe nycthémérale normale, en l'absence de HB 419).

Cette interprétation est en accord avec les constatations histologiques que nous avons pu effectuer sur le pancréas prélevé au cours de biopsies:

Deux mois après l'arrêt du traitement d'une durée de 5 mois par le HB 419, nous avons constaté que le pancréas contenait de nombreux îlots de Langerhans néoformés s'infiltrant entre les masses acineuses; ils présentaient des interdigitations comparables à celles que l'un de nous a précédemment décrites [25]. De nombreux petits îlots riches en saccules renfermant des granules d'insuline étaient également présents. Cela signifie que les effets histologiques bêtacytotrophiques ne sont pas fugaces, mais persistent pendant plusieurs mois après l'arrêt de l'administration du produit.

3. Nous avons également recherché quelle quantité d'insuline était nécessaire pour reproduire chez le chien normal les mêmes effets sur la glycémie que ceux provoqués par le HB 419. Pour réaliser cette expérience nous avons utilisé une méthode expérimentale et un appareillage conçus dans notre laboratoire. Lors d'un premier temps opératoire, deux cathéters ont été introduits chez un chien anesthésié, dans deux veines superficielles, en l'occurrence dans la veine céphalique des deux membres antérieurs. L'animal était ensuite placé dans une cage munie d'un dispositif spécial. Celui-ci permettait chez le chien éveillé, menant une vie normale, de perfuser d'une manière continue une solution d'insuline dans une des veines et de prélever simultanément du sang dans la veine contralatérale afin d'effectuer des dosages de glycémie de façon continue à l'Auto-Analyseur Technicon. Des dosages d'insulinémie pouvaient également être faits.

Nous avons ainsi établi que pour maintenir $(18 \mathrm{~h}$ après le dernier repas) un abaissement glycémique de 30 à 35 p. 100, il fallait administrer d'une manière continue 0.85 unités d'insuline par kg et par $24 \mathrm{~h}$, soit 12.75 unités par $24 \mathrm{~h}$ pour un chien de $15 \mathrm{~kg}$.

L'aire d'hypoglycémie ainsi obtenue pendant $24 \mathrm{~h}$ est comparable à celle de la courbe nycthémérale réalisée par le HB 419 chez l'animal traité chroniquement depuis 1 mois par ce produit.

Ceci semble signifier qu'en un mois, le pancréas de ces animaux a sécrété 382.5 unités d'insuline en plus de sa sécrétion normale, soit ,en 4 mois, 1530 unités.

Le HB 419 aurait done apparemment provoqué en 4 mois la synthèse et la sécrétion de 1530 unités d'insuline endogène, en plus des quantités normalement sécrétées.

Il est logique de penser que ce résultat est la conséquence de l'action bêtacy totrophique du HB 419 (augmentation du nombre des îlots, des cellules bêta et 
de la synthèse de l'insuline) et de l'action stimulante bêtacytotrope que ce produit exerce sur la sécrétion d'insuline endogène.

Nous derons cependant souligner que le calcul cidessus n'a qu'un caractère approximatif. En effet en plus de son action insulino-sécrétrice directe sur les cellules bêta des îlots de Langerhans, le glibenclamide, d'une part potentialise les effets hypoglycémiants de l'insuline et, d'autre part, possède peut-être d'autres effets de moindre importance qui peuvent éventuellement s'ajouter pour constituer l'action hypoglycémiante totale du sulfamide.

Rappelons que nos expériences antérieures réalisées sur le chien privé chirurgicalement des $9 / 10 \mathrm{e}$ du pancréas avaient montré que l'administration de sulfamide hypoglycémiant (chlorpropamide) pendant plusieurs mois et même pendant plusieurs années ne provoque pas l'épuisement des cellules bêta des îlots de Langerhans, et retarde d'une manière considérable l'installation du diabète sucré exerçant ainsi une remarquable action préventive $[22,44,32,42,23,36,33,25,37,39]$.

Rappelons que dans ces expériences nous avions conservé chez 8 chiens, un dixième du pancréas autour du point d'attachement du canal de Wirsung sur le duodénum; nous avons ainsi constitué un petit pancréas. Leur nourriture était constituée par de la viande crue (30 g par $\mathrm{kg}$ et par $24 \mathrm{~h}$ en deux repas). Sur ces 8 chiens, les quatre témoins sont devenus diabétiques dans une période de 17 mois en moyenne après l'opération. Quatre autres ont été traités par du chlorpropamide (25 mg/kg/jour per os). Trois d'entre eux n'étaient pas diabétiques 32 mois après l'opération; ils moururent de complications post-opératoires consécutives aux biopsies pancréatiques auxquelles ils furent soumis. Le quatrième n'était pas encore diabétique 72 mois après la pancréatectomie subtotale. Chez les quatre animaux traités, le chlorpropamide a donc manifesté une influence retardatrice sur l'apparition du diabète.

Dans ces expériences nous avions également constaté l'abaissement de la glycémie à jeun ainsi que celui de la courbe nycthémérale de la glycémie. On trouvera une analyse des mécanismes impliqués dans cette action préventive et retardatrice dans l'une des publications de l'un de nous [25].

Vis-à-vis de l'action néogénétique sur les îlots de Langerhans et les cellules bêta (action bêtacytotrophique), le glibenclamide paraît done se comporter comme les autres sulfamides hypoglycémiants qui l'ont précédé dans l'expérimentation animale et humaine.

En conséquence, il nous paraît raisonnable de proposer l'utilisation de cette substance en vue d'essais cliniques destinés à prévenir ou retarder l'installation du diabète sucré chez les sujets humains prédisposés à cette affection. Dans ces cas, des doses infiniment moindres que celles utilisées pour d'autres sulfamides hypoglycémiants devront être employées.

Il convient cependant d'insister d'une part sur le fait que le traitement préventif et prophylactique du diabète par les sulfamides hypoglycémiants doit être institué aussi tôt que possible, c'est-à-dire pendant les premières phases de l'état prédiabétique. $D^{\prime}$ autre part, sur le fait que pendant ce traitement un régime alimentaire adéquat doit être appliqué et maintenu.

C'est à ces conditions qu'un résultat profitable sera susceptible d'être constaté dans un nombre important de cas.

Les conclusions que l'on peut tirer des expériences que nous avons effectuées sont les suivantes:

1. Chez la souris le HB 419 administré chroniquement stimule la néogénèse des îlots de Langerhans et des cellules bêta. Il est done doué de l'action bêtacytotrophe.

2. Chez le chein normal l'administration chronique de HB 419 a pour effet d'abaisser la glycémie à jeun et la courbe de la glycémie nycthémérale (dosée pendant les 24 h de la journée).

L'expérimentation effectuée suggère que sous l'influence de l'administration prolongée de HB 419, la quantité d'insuline endogène synthétisée et sécrétée en plus de celle qui est normalement synthétisée et sécrétée est considérable.

Le HB 419 possède donc toutes les actions précédemment attribuées aux sulfamides hypoglycémiants antérieurement décrits, mais leur manifestation nécessite des doses infiniment moindres.

Cette activité considérable qui se manifeste à faible dose, constitue à notre avis un des avantages essentiels de ce produit pour des traitements de longue durée dans lesquels il est important de réduire au maximum l'apport pondéral médicamenteux.

\section{Bibliographie}

1. Aluic, R., M.M. Mariant et A. Loubatieres: Importance de l'état des éléments figurés du sang et en particulier de celui des globules rouges sur les valeurs du glucose sanguin mesurées par l'Auto-Analyseur Technicon. Path. Biol. 13, 506-511 (1965).

2. Ashworth, M.A., and R. E. Harst: Some effects of $\mathrm{BZ} 55$ (carbutamide) on the growth of the islets of Langerhans. Canad. med. Ass. J. 74, 975 (1956).

3. Bänder, A., A. Haussler u. J. Soholz: Ergänzende pharmakologische Untersuchungen über Rastinon. Dtsch. med. Wschr. 82, 1557 (1957).

4. BLOodworth, J.M.B., Jr.: Morphologic changes associated with sulfonylurea therapy. Metabolism 12, $287-301$ (1963).

5. Bunnag, S.C., N.E. Warngr, and S. Bunnag: Effect of tolbutamide on postnatal neogenesis of the islet of Langerhans in mouse. Diabetes 15, 597-603 (1966).

6. Creutzfeidi, W., u. G. Geginat: Glukosetoleranz und Inselregeneration bei teilpankreatektomierten Ratten unter ACTH und langfristiger Behandlung mit $\mathrm{N}$-(4-methylbenzolsulfonyl)N-butylharnstoff. Arzneimittel-Forsch. 8, 464-469 (1958).

7. Davidson, J.K., and R.E. HAIST: Islet weight studies in rats treated with tolbutamide. Diabetes 11 suppl., $115-120(1960)$.

8. Fruteau de Laclos, C., et A. Lotbatreres: Effets des arylsulfonamides hypoglycémiants sur la structure histologique du pancréas. Bull. Ass. Anat. 106, $261-$ 278 (1960). 
9. Gepts, W.: Histopathologie des îlots de Langerhans après traitement oral du diabète. In Oberdisse, $K$. and Jahnke, K. ed. International Diabetes Federation, 3rd Congress, Düsseldorf, p. 255. Stuttgart: Georg Thieme Verlag 1958.

10. Grimelius, L., G. T. Hultquist, J. Theorelret, and L. WrnbladH: Studies on islet tissue transplants in the anterior chamber of the eye in rats. In "The structure and Metabolism of the pancreatic islets", edit. by S.E. Broutn, B. Heltman et H. KnUtson, p. 173. Pergamon Press 1964.

11. Hars', R. F. : Islet cell function. Ann. N.Y. Acad. Sci. 82, art. 2, 266-286 (1959).

12. HAIST, R.E., and E.J. PUGH: Volume measurement of the islets of Langerhans and the effects of age and fasting. Amer. J. Physiol. 152, 36-41 (1948).

13. HALES, C.N., and P.J. RANDLT: Immunoassay of insulin with insulin-antibody precipitate. Biochem. J. 88, 137 (1963).

14. Kracht, J.: Experimentelle Morphologie des Inselorgans unter BZ 55, D 860 and IPTD. Medizinische $12,525-528(1959)$.

15. - B. KröneR, L. Von Hout, u. C. Von Holt: Zunahme von Plasmainsulinaktivität und B-Zellenmitosen nach $\mathrm{N}$-(4-methyl-benzolsulfonyl)-N-butylharnstoff. Naturwissenschaften 44, 16-17 (1957).

16. Lambert, A., D. Vecchio, A. Gonet, B. JeanrenAUD, and A.E. RENOLD: Organ culture of fetal rat pancreas: Effects of tolbutamide, glucagon and other substances. In "Tolbutamide after ten years", Brook Lodge Symposium, mars 1967, Excerpta Medica Foundation, International Congress Series no $149-$ 61-82 (1967).

17. Lever, J.D., M.K. Jlacock, and F.G. Young: The production and cure of metahypophyseal diabetes in the cat: a biochemical and electron microscopical study with particular reference to the change in the islets of Langerhans of the pancreas. Proc. roy. Soc. (Biol.) 154, 139-150 (1961).

18. Lw Compte, P.M., and J.C. MrRRIam: Mitotic figures and enlarged nuclei in the islands of Langerhans in man. Diabetes 11, 35-39 (1962).

19. Loubatieres, A.: Physiologie et Pharmacodynamie de certains dérivés sulfamidés hypoglycémiants. Contribution à l'étude des substances synthétiques à tropisme endocrinien. Thèse de Doctorat ès Sciences Naturelles, $n^{\circ}$ 86. Montpellier: Causse, Graille et Castelnau 1946.

20. - The mechanism of action of the Hypoglycemic Sulfonamides: a concept based on investigations in animals and in human beings. Ann. N.Y. Acad. Sci. 71, $192-206$ (1957).

21. - Pharmacodynamie des arylsulfonamides hypoglycémiants. Analyse de leur mécanisme d'action chez l'animal ou chez l'homme à l'état normal ou diabétique. III Congress of the International Diabetes Federation, Düsseldorf, OBERDISSE, K. et K. JAHNKE edit., p. 279-286. Stuttgart: Georg Thieme Verlag 1958.

22. - Experimental studies for the tentative use of hypoglycaemic sulphonamides as prophylactic agents against diabetes. Proc. roy. Soc. Med. 53, 595-599 (1960).

23. - Pharmacological action of the hypoglycemic arylsulphonamides upon the histophysiology and the physiology of the beta cells of the islets of Langerhans of the pancreas. In: "Pharmacological control of release of hormones including antidiabetic drugs", First International Pharmacological Meeting, Stockholm, Pergamon Press, edit. 1, 47-89 (1962).

24. - Déterminisme de l'action trophique que les sulfamides hypoglycémiants exercent sur l'appareil cel-
Iulaire bêta des îlots de Langerhans du pancréas. Arch. int. Pharmacodyn. 140, 127-137 (1962).

25. - Effects of prolonged administration of hypoglycaemic sulfonamide (chlorpropamide) upon the subtotally pancreatectomized dog. In "The structure and Metabolism of the pancreatic islets", Wenner-Gren Center, International Symposium Series, Vol. 3, edit. by S.E. Brolin, B. Hellman and H. KNutson, p. 437-450. Pergamon Press 1964.

26. - P. Bouyard et C. Fruteau de Laclos: Analyse du mécanisme de l'action curatrice que le p-aminobenzène-sulfamido-isopropylthiodiazol exerce sur le diabète sucré expérimental provoqué par l'alloxane. C.R. Acad. Sci. 241, 515-516 (1955).

27. - - - Actions du para-aminobenzène-sulfamidoisopropylthiodiazol sur la glycémie, la structure des îlots de Langerhans et le métabolisme de l'animal normal ou diabétique. C.R. Soc. Biol. 149, 1642-1646 (1955).

28. - - - Action curatrice du p-aminobenzène-sulfamido-isopropylthiodiazol sur le diabète sucré expérimental. Analyse du mécanisme d'action de cette substance. Le Diabète 4, 38-40 (1956).

29. - - - et A. SASSINT: La "guérison》spontanée du diabète sucré méta-alloxanique provoquée chez le chien par les sulfamides hypoglycémiants. Conditions expérimentales et caractères. C.R. Soc. Biol. 151, $2179-2182(1957)$.

30. - et C. FrUteaU de Laclos: Preuves histologiques de l'action bêta-cytotrope pancréatique des arylsulfonamides hypoglycémiants. C.R. Soc. Biol. 152, $1760-1763$ (1958).

31. - - M.H. Houareau et R. Atrio: Etude quantitative de la néogénèse des îlots de Langerhans du pancréss, provoquée par un sulfamide hypoglycémiant, le tolbutamide. C.R. Soc. Biol. 157, 16521654 (1963).

32. - - M.M. Mariant et A. SAssine: Sur l'importance du maintien des fonctions exocrine et endocrine $d u$ pancréas pour la bonne tolérance de certains sulfamides et la manifestation de leur action hypoglycémiante et antidiabétique. C. R. Soc. Biol. 154, 173-175 (1960).

33. - - - Effets de l'administration prolongée de sulfamide hypoglycémiant sur le chien complètement dépancréaté, greffé ou porteur d'un "petit pancréas» conservé «in situ». Le Diabète 11, 25-33 (1963).

34. -, et M.M. MarianI: Etude pharmacologique et pharmacodynamique d'un sulfonylurée hypoglycémiant particulièrement actif, le glybenzcyclamide. C. R. Acad. Sci. 265, série D, 643-645 (1967).

35. - - et R. Atric: Actions respectives ou simultanées du tolbutamide et du diazoxide sur le développement des îlots de Langerhans. C.R. Acad. Sci. 265, série D, $2127-2131$ (1967).

36. - - - et C. Fruteau de Laclos: Action antidiabétique d'un sulfamide hypoglycémiant (chlorpropamide) sur le diabète latent expérimental du chien. C.R. Soc. Biol. 157, 2247-2249 (1963).

37. - - - Action prolongée du chlorpropamide sur le diabète latent provoqué chez le chien par pancréatectomie subtotale. Ve Congrès de la Fédération Internationale du Diabète, Toronto. Excerpta Medica International Congress Series 74, Abst. 162 (1964).

38. - - - et M.H. Houareau: Effets sur le développement et la néogénèse des îlots de Langerhans du pancréas, d'un sulfamide hypoglycémiant particulièrement actif : le glibenclamide. C.R. Acad. Sci. 267, Série D, 236-239 (1968).

39. - - - et J. TAYLOR: Le traitement préventif du diabète sucré par le chlorpropamide. Etude chez le chien présentant un diabète potentiel expérimental. C.R. Soc. Biol. 159, 1799-1801 (1966). 
40. - - J. Chapat, G. Rrbes et H. De Malbosc: Etude expérimentale du glibenclamide (HB 419). Com. $\mathrm{n}^{\circ} 89$, IVe Congrès de l'Association Européenne pour l'Etude du Diabète, Louvain 22-24, juillet 1968.

41. - - G. Ribes, H. DE Malbosc et J. ChapaI: Etude expérimentale d'un nouveau sulfamide hypoglycémiant particulièrement actif, lo HB 419 ou glibenclamide. I. Action bêtacytotrope et insulino-sécrétrice. Diabetologia 5, 1-10 (1969).

42. - - A. SASSINE et C. FruTteaU DE LaClos: Nouvelles observations concornant les effets de l'administration pendant vingt mois de chlorpropamide au chien sensibilisé au diabète par dépancréatation subtotale. $\mathrm{C}$. R. Soc. Biol. 155, 335-338 (1961).

43. - A. Sassine, C. Fruteau de Laclos et P. Bouyard : Action des sulfamides hypoglycémiants sur la croissance du rat blanc et l'histologie du pancréas. C.R. Soc. Biol. 151, 957-959 (1957).

44. - - M.M. Mariani et C. Fruteau de Laclos: Action antidiabétique des sulfamides hypoglycémiants chez le chien privé des neuf-dixièmes du pancréas. C.R. Soc. Biol. 154, 1475-1478 (1960).

45. - J. Taylor, M.H. Houareat et R. Atric: Activité néogénétique pour les îlots de Langerhans du benzène-sulfamido-tertiobutyl-thiodiazol. C.R. Soc. Biol. $159,156-158$ (1965).

46. SAK, M.F., I. MACCHI, and S.B. BEASER: Structural and functional characteristics of neonatal pancreas homografts in alloxan diabetic golden hamsters. Diabetes 15, $51-58(1966)$.

47. V \#CCHIo, D., et A.E. Gonet: Culture d'organe de pancréas foetal de rat: I. Effets du glucose, d'autres composants du milieu de culture et d'un sulfamide hypoglycémiant. Helv. physiol. pharmacol. Acta 25, 103-122 (1967).

48. VeCCHro, D., A. LuYokx et A. E. Renold: Culture d'organe de pancréas foetal de rat. II. Effets du glucose, d'un sulfamide hypoglycémiant et du glucagon sur la libération de l'insuline. Helv. physiol, pharmacol. Acta 25, 134-146 (1967).

Prof. Dr. A. Loubatières

Faculté de Médecine

Institut de Biologie

Boulevard Henri IV

Montpellier, France 